\title{
The Design of Spaces for Young Children and Preschool in Emergency
}

\author{
By Nicoletta Sorrentino*
}

\begin{abstract}
The paper aims to show how to develop and treat spaces devoted to childhood and the restarting of teaching activities after disasters, in situations of emergencies, particularly reflecting the needs of early childhood age. This choice is motivated by the fact that this early stage of age requires diverse and adequate devices, especially for what concerns the constitution of the environment where they live and experience extraordinary conditions. The essay will present, through quantitative evaluation of the impact of disasters on minors, the significance and the consequences of traumatic events on children, as expressed in their way of behaving and reacting both personally and within the relation with the community. The research methodology, progressing from a general presentation of the psychological facets and focusing on the dynamics of relationships with adults and other children, follows the narrative of the features of the correct preschool environment. As a result, the research depicts the peculiarities of proper strategies for mitigation and intervention in the occurrence of disasters, as how expressed by NGOs. Furthermore, the essays tend to become more technical, examining the state of the art and indicating good practices of design for childhood purposes and preschools; on the other hand, less addressed designs, actually widely used will be compared. In the end, the paper will show some concept designs, applicable to different contexts and landscapes, on the Italian territory, that for their inner peculiarities could be used as early age children school. On the basis of what will be presented in the research, a design process has been constituted for achieving valuable answers, coherent with the theme under examination, also applicable once stopped the emergence and in extraordinary conditions. The attention focuses on the interior and furniture design and its setting, that in most cases are not enough cared, while for the external housing structures the concepts offer different solutions, also considering products already part of the mass production.
\end{abstract}

\section{Introduction}

This research began in 2012 and was the subject of the author's graduate studies for the Architecture Degree at the University of Genoa, under the guidance of Prof. Carlo Vannicola and Dr Luisa Chimenz.

The initial idea was born on one hand by some observations on the current historical phase and will be explained below. According to the reports and the latest information of Save the Children and other agencies among the most reliable non-governmental organizations (NGOs), a significant number of children every day, are forced to deal with the effects of natural disasters or armed conflict. So, they lose the opportunity to attend school, educate and socialize with peers, and are at risk of ill-treatment and abuse. Moreover, between 2009 and 2012 they took place in Italy several natural disasters (earthquakes and floods). Among the several

\footnotetext{
*Tutor/Assistant, Department Architecture and Design, Università degli Studi di Genova, Italy.
} 
consequences of these events, there was the suspension of school activities for children of the affected areas, as school buildings were condemned or at worst, destroyed.

Thus, faced with this situation, a simple question occurred. How can architecture and design do their part and meet the challenges that these conditions present to us, if they can do it? Observing the means of intervention most widely implemented in these circumstances, emerged some critical aspects that are not addressed, probably because they are not perceived as a priority yet. Furthermore, examining the current responses in terms of structures and equipment, very critical issues have been highlighted.

Then, what is the contribution that architects and designers can give, by virtue of their skills? How could they improve the solutions put in place, so that they are more responsive to the needs of children, not only from the functional point of view but also alleviating the difficulties in such critical moments, providing welcoming, attractive and safe environments?

The paper aims to describe the main stages of the research originated with these questions, clearly showing the logical path followed from the identification of the problems to the solutions proposed, taking into account the various factors at play.

The main new feature of this research lies in its multidisciplinary, in the effort to outline an experimental approach to design, with the intention to show how to develop and treat spaces devoted to childhood and to the restarting of teaching activities after disasters.

\section{Methodology}

The research methodology starts from a general presentation of the psychological facets and the dynamics of relationships with adults and other children and then is progressing with the narrative of the features of the correct preschool environment.

Through a brief quantitative evaluation of the impact of disasters on minors, they have been presented the significance of traumatic events and the way the children react to their consequences, both personally, and within relation with the community.

As a result, they have been depicted the peculiarities of proper strategies for intervention in the occurrence of disasters. According to NGOs' indication, it is analysed the state of the art, in a comparison between the good practices of design for childhood purposes and preschools, and less addressed but actually, widely used solutions.

Finally, the paper outlines the design process built on these considerations and shows some concept designs, applicable to different contexts and landscapes of the Italian territory and inner peculiarities could be used as a school for children of an early age. 
Children between the Age of 3 and 6

Above all, it has been considered that, before examining the reactions of children to disasters, it should be convenient to know their behaviour in normal conditions.

At this early stage of age, children grow up facing many changes, so it is very important for them to have the references by which to understand reality.

In this process of building knowledge, a few elements are of particular importance for the final purpose of this research, presented as follows.

Routines are essential socio-cognitive and emotional anchors, as they help the child to deal with ambiguous aspects and unexpected problems or conflicts, in the course of daily life.

Being part of a social group gives children a usual basis for sharing moments and experiences with each other and contributes to building a sense of belonging to a community.

New things are often perceived as a source of fear at first, so children have to be helped to transform back into a normal atmosphere what at first is unfamiliar.

Peer relationships and friendships are kinds of bonding that bring to the creation of a commonly shared world. They are also a powerful force to overcome the negative emotions, by virtue of which children develop solidarity and empathy.

Playing is the main activity of children, whereby they extend their experience of the surrounding reality and deepen their empirical knowledge. Playing is also a cathartic experience, by means of which children can re-balance their emotions. Finally, while they play through the simulation, children train themselves to be sons, parents, teachers, doctors and so on, and they make own roles and rules.

The peer culture is the element that allows children to not feel alone in acquiring and interpreting the adult world. It is a stable set of activities or routines, objects, values and concerns that children produce and share in interaction with each other. It is highly symbolic and has its origin in the usual interests of children (fairy tales, cartoons, toys) and represents their attempt to make sense of things. Another central aspect of the interaction between peers is to challenge the authority of adults. Sharing the prohibited behaviour with peers means mainly to give a common significance to the violated rule. In the peer culture, finally, they are incorporated also real fears, identifying strategies to address them and process them through the control in the game routines and rituals.

\section{Main Features of Preschools}

In the same way, as done examining the behaviour of children, the choice has been to consider the main features of preschools in normal conditions, before studying the emergency context.

The kindergarten is the place where children often experience the first contacts with peers and where they establish new educational relations with adults other than parents. Preschool becomes a physical and temporal space called "educational environment", that is intended to lead boys and girls, each in their own time, to the improvement of their identity, autonomy, competence and sense 
of participation and citizenship. "If the environment contributes to the growth and education of the child, it is inevitable to wonder about its nature; if you take pleasure entering in it (adults and children), and if you experience satisfaction being there every day.

If this is the feeling you have, it can deduce that it is a nice, pleasant, friendly, a facilitating environment that supports the child in the fundamental tasks of his growth: attainment, building personal strategies of exploration, knowledge, experience and appropriation." 1

As school at first could not be perceived positively and so it could not be accepted, it is particularly important the scan of various time steps, in order to enhance the organizing function of children's thinking. Thus, the establishment of new routines will help the child becoming familiar with the new environment and to accept it, during the separation from parents. "The routines, then, run the time of life at school" and "[...] return the child to a sense of continuity and stability in change," 2 becoming symbolic representation not only of the individual but of the identity of the whole group. Finally, the physical characteristics of the school environment which is one of the most relevant aspects from the point of view of architecture and design, and of main importance in the development of this research. It can, in turn, affect the climate of the educational environment, facilitating or hindering learning. In this sense, it is relevant to this issue the Reggio Emilia Approach, "an educational philosophy based on the image of the child, and of human beings, as possessing strong potentials for development and as a subject of rights who learns and grows in the relationships with others,"3 as written on the website of Reggio Children organisation. In this unique, educational vision, the setting of school spaces takes on a special significance. In fact, all properties of the school environment are considered important in connote, positively or negatively, the quality of life that takes place in them, so their performance must be studied very carefully. According to Rosanna Bosi, "Space is a fundamental educational vehicle. It is never a neutral container, and it has its own specific language," so its material design is a decisive step in the educational action, having its goal as the creation of an environment at the same time stimulating and reassuring.

\section{Children and the Emergency}

In the research phase, it was particularly difficult to raise disaggregated statistical data, explicitly referred to the involvement of children in crisis situations.

However, this does not prevent to outline a picture of the extent of the situation, both globally that in the narrower field of the Italian territory.

In fact, it is sufficient to refer to the main humanitarian agencies, to get an idea of how many children must deal daily with the consequences of natural disasters or conflicts, and socio-political instability.

\footnotetext{
1. R. Bosi, La Cura Nella Scuola dell' Infanzia (Rome: Carocci, 2007), 72.

2. Ibid, 87.

3. Refer to http://www.reggiochildren.it/identita/reggio-emilia-approach/?lang=en.

4. Bosi, La Cura Nella Scuola dell' Infanzia, 2007, 77.
} 
"Natural disasters and armed conflict have marked human existence throughout history and have always caused peaks in mortality and morbidity. But in recent times, the scale and scope of these events have increased markedly. Since 1990, natural disasters have affected about 217 million people every year, and about 300 million people now live amidst violent insecurity around the world.",

Referring to the current situation, and to the most up-to-date data related to the very latest events, for example, we find that in Haiti up to 130,000 children were estimated to be out of school following the Hurricane Matthew (2016). ${ }^{6}$

In Syria, six years since the war began, 5.8 million children still live under shelling and 3 million of them, who are now six years old, have never known anything but war. ${ }^{7}$

Finally, remarking the Italian case, the seismic crisis occurred between August and October 2016 hit more than 30,000 people living in a complex territorial situation and settlement. ${ }^{8}$ These examples certainly cannot be considered exhaustive, however, they depict significantly the general framework, highlighting how the problem of emergencies is globally spread.

\section{Definition of "Traumatic Event" and Consequences Concerning Individuals and Community}

The word trauma usually indicates an "emotional shock following a stressful event or a physical injury, which may lead to long-term neurosis." "9

"A traumatic event or situation creates psychological trauma when it overwhelms the individual's ability to cope, and leaves that person fearing death, annihilation, mutilation, or psychosis. The individual may feel emotionally, cognitively, and physically overwhelmed."10

Broadly speaking, the trauma can be identified in an experience of particular gravity, such as to impair the sense of stability and continuity of physical or mental health of a person.

Disasters can be considered as traumatic events, for their power to exceed the human experience and to induce stress reactions in anyone. People who are facing the consequences of any type of disaster live a sudden and disruptive fragmentation of their existential continuity, which can put a strain on their capacity to adapt their psychological health.

This could be even more difficult for children, who have still immature ability to cope with the difficulties. The perception of danger often causes fear, a sense of helplessness, in front of death and destruction. Confusion and disorder are manifested in increased vulnerability.

5. See J. Leaning and G. S. Debarati, "Natural Disasters, Armed Conflict, and Public Health," New England Journal of Medicine 369, no. 19 (2013): 1836.

6. Source Save the Children International. See https://bit.ly/2tCMYOL.

7. Source Save the Children Italy. See https://bit.ly/2mro7I0. 2UaDLbE.

8. Extrapolated Data from a Custom Research in EM-DAT Database. See https://bit.ly/

9. Definition from the Online Version of Oxford Dictionaries (retriev.) https://bit.ly/2Snm A5h. [Accessed 03/05/2017].

10. See E. Giller, What is Psychological Trauma (Brooklandville, MD: Sidran Institute, 1999). 
This mix of counter emotions is frequently difficult to manage and could add a dangerous sense of distrust of adults, if the facts have been deliberately caused by humans, such as war or terrorist attacks.

Even if children could not find themselves to personally experience the extreme consequences of the calamity, the impact of disasters on their daily life generates immediate instability and uncertainty. They may have to suddenly adapt to makeshift accommodations, or it might not be possible to go to school, to play sports and to meet with friends.

The results could be a strong break between before and after in the lives of involved children, in their established routines, and a new daily life may have to be built overnight, in extreme cases without the prospect of a return to previous normality.

It is evident that the response of the child is influenced by the one of the adults: as much as they demonstrate grasp of the situation and control, the material and emotional adaptation of children increases.

"Among the survivors of a disaster, the vast majority of people [...] shows signs of stress, so that stress reactions are considered a normal reaction to abnormal events." 11

The individual response depends on many different factors, starting from the type of experience and its severity. For children, they mainly rely on aspects such as proximity to the place and awareness of the disaster (evidently related to the age), having been injured or in mortal danger (real or perceived), or have even lost family and friends. Something to be considered too, is the loss of material goods, the intensity and duration of the disruption of his life and the reactions of those around them. It is possible to observe several typical consequences in children:

- the increased dependence on adults;

- the occurrence of nightmares and other sleep disorders;

- the regression in the development of evolutionary stages;

- the outbreak of specific fears in relation to objects that evoke the experience;

- the repetitive representation of the disaster in post-traumatic play. ${ }^{12}$

The traumatic impact of the event generates a response not only at the individual level, but also a community reaction. The two things are tangled and affect each other.

In fact, the relationship between the individuals and their own communities will change significantly during the post-emergence phases. Initially, there are stronger links within civil society, with a big boost to solidarity and to mutual aid. Later, the situation can consolidate, especially in the long term. This may cause the opposite effect, leading to the creation of groups and subgroups with consequent conflicts for receiving aid and housing. These dynamics negatively influence the sense of community and can be hardly recovered once the

11. G. Lo Iacono and L. Ranzato, "Aiutare i Bambini Sopravvissuti a Calamità: Indicazioni per Insegnanti e Genitori," Psicologia e Psicologi I, no. 3 (2001): 2.

12. Ibid, 5. 
emergency has stopped.

\section{Child-Friendly Spaces as a Paradigm of Response}

The Child-Friendly Spaces (CFS) are intervention instruments provided by various humanitarian organizations, as a response in the short- and medium-term to the effects of a crisis.

"The purpose of CFSs is to support the resilience and well-being of children and young people through community organized, structured activities conducted in a safe, child-friendly, and stimulating environment." 13

They are often arranged in tents or other provisional structures, in the aim to offer a child centred environment. They include the possibility of tailor-made play areas for different ages, education, health and psycho-social support services.

As expressed by the Global Protection Cluster in cooperation with Global Education Cluster, INEE and IASC, "A coordinated, inter-agency, and multisectorial approach to CFSs is needed to address the needs of the affected population and achieve program consistency, quality, and sustainability," ${ }^{\prime 4}$ in the aim to ensure the effective functioning of this kind of measures.

Thus, in establishing CFSs it is of paramount importance operating appropriately to the contexts and the culture. It should take a coordinated, interagency and multi-sectorial approach. CFSs should be used as a means of mobilizing the community and be the most inclusive and non-discriminatory; to ensure security and safety; to be stimulating, participatory and supportive environments. 15

\section{The Children's Behaviour and the School's Role}

Belonging to a group or community, as well as the dynamics within them, influence the experience of the individual already in normal conditions. In the occurrence of extraordinary situations, it is of even greater importance for children.

Thus, it emerged that school can play a positive role at several levels, with beneficial effects both on the individuals and on the whole community. They can be grouped into three categories, as follows.

\section{Physical Aspects}

School provides safe spaces where play and spend time with friends. The school environment is an easily recognisable place that can be uniquely identified by the youngest, helping them to establish a new benchmark in the construction of everyday life in the camp.

The spatial arrangement and the organization of the activities can significantly

13. See Global Protection Cluster. Guidelines for Child Friendly Spaces in Emergencies (Global Education Cluster, INEE \& IASC, 2011), 2.

14. Ibid, 4.

15. Ibid, 3 . 
contribute to adaptation to the new situation, recalling and re-enacting well-known dynamics.

\section{Psychological Aspects}

The creation of special conditions and attitudes in respect of affected children can lead to re-establish their confidence and security.

Children may share with peers their impressions about the new situation and so find reassurance from the bottom up, resulting in a positive effect of brightening.

Also, thanks to its physical features, the school can support the restoration of control and predictability of the events, a requirement of main importance for children.

\section{Social Aspects}

The school has a role of primary importance, at a double level: through the involvement of individuals, it leads to benefit the whole community.

In the extremely informal emergency context, some prejudices can lapse, so who had been hitherto excluded from the group could start to join it.

As the group is such a significant element of socialization, hold together the existing classes, as far as possible, is a factor that should not be underestimated.

They contribute to the consolidation of the routine not only time and place where the group meets, but the same people who belong to it and share them together.

Furthermore, through education, it is possible for a community to keep its identity and the maintenance and development of the mother tongue. Addressing identity dispersion generated by the logic of "non-place", and promoting an attitude of resistance, keeps the bond of the community with its own history. ${ }^{16}$

According to Maria Vittoria Isidori and Alessandro Vaccarelli, it is so highlighted that "Especially in chronic situations, provide education allows a population of not lowering the standards achieved and to work on an idea of future projects, involving at the same time individuality and sense of community."17 Moreover, as stated by Vanna Iori, "In moments of crisis, [...] the essential task of educational institutions is to restore the temporal coordinates, within which unravels the existence of a person, renovating the symbolic, intrapsychic space and relational, which makes everyday life, in order to reconstruct and reinterpret the previous life experience and the current one." 18

In conclusion, not including the family, school is the only institution capable

16. For a more complete discussion on this topic, refer to M. V. Isidori, "Principali Criticità della Pedagogia e della Didattica dell' Emergenza," Studi sulla Formazione 13, no. 1 (2010): 159160 .

17. See M. V. Isidori and A. Vaccarelli, Pedagogia dell' Emergenza/Didattica nell' Emergenza. I Processi Formativi nelle Situazioni di Criticità Individuali e Collettive (Milan: Franco Angeli, 2013), 155.

18. V. Iori, Lo Spazio Vissuto: Luoghi Educative e Soggettività (Florence: Nuova Italia, 1996), quote in Isidori, "Principali Criticità della Pedagogia e della Didattica dell' Emergenza," 135. 
of restoring a frame that literally "bracket" the life of children, making easier for them to deal with the consequences of disasters and emergency situations.

\section{Design for Emergency School Architecture}

Regarding the strategies of intervention from a more technical point of view, the research had examined the state of the art, depicting a general overview of the situation.

On one hand, they arise some models that could be considered as best practices, even if sometimes they were not initially designed for this purpose.

On the other, they have been described as cases not free from problematic issues, as they however, represent the most widely used solutions.

From a first schematic analysis, it appears that in most cases the recovery of school activities is initially implemented in tents, equipped with retrieved or makeshift minimal supplies. After, it transfers to temporary structures, half the time comparable to containers. They certainly offer greater comfort than the tents, but however present considerable critical aspects.

Broadly speaking, the issue seems to be focusing more on the outer shell, however with little consideration of what and how that case will contain. As a result, many times the arrangements appear inappropriate and seem almost left to chance and even more boosting the state of insecurity. The research gives a brief review of projects, organized into two sections, Architecture and Partitions and furniture. They include mobile units and self-construction architectures, as well as ordinary furniture and temporary solutions. Due to space constraints, not all projects originally examined can be here included, so they will be presented two models for section, and compared both "good practice vs improvable model".

\section{Architecture: Hualin Temporary School vs M.U.S.P. Project}

The Hualin Temporary School was realized in the aftermath of the earthquake in Sichuan (China) in 2008. It was designed in a project of cooperation between Chinese and Japanese universities, under the direction of Shigeru Ban.

The Inter-University group, composed mostly by students and volunteers, designed and built in about forty days three buildings, for a total of nine classrooms. The project uses the same technology developed by Shigeru Ban for the Paper Log Houses, ${ }^{19}$ introducing some changes in statics and building schemes.

The bearing structure is constituted of four arches, realized with paper tubes connected to each other by wooden joints. They compose a frame, to which are added sliding doors and PVC panels, while the roof is made of corrugated polycarbonate sheets. The high potential value of this project lies mainly in the speed of realization obtained thanks also to dry assembly. Despite its simplicity, it also offers an essential space but of high architectural quality.

19. The Paper Log Houses were developed after the earthquake in Kobe, Japan, in 1995. For further information refer to http://www.shigerubanarchitects.com/works/1995 paper-log-house -kobe/index.html. 
M.U.S.P. is the acronym of the Italian expression for "solid temporary school modules." 20 They are containerized prefabricated structures, suitable to be placed in a number of contexts, up to 1,500 meters above sea level.

They consist of a metal bearing structure with infill insulated panels, which constitute standard units. They can be used individually or combined with each other, according to various distribution patterns, in order to obtain the solution best fitting the users' needs.

Alongside the undeniable advantages (availability, modularity, easy carriage and assembly), however, they present several problematic issues, especially concerning the thermal comfort and the limited possibilities of customization of the aesthetic appearance. In the long term, they may affect the physical and psychic well-being of the users. Nevertheless, they represent the solution of wider use and not only in Italy.

\section{Partition and Furniture: Leafbed vs Education in a Box}

Leafbed is a product of the French company Leaf Supply, which designs and realizes cardboard furniture for humanitarian purposes. ${ }^{21}$

It is a patented camp bed, made up of cardboard boxes folded in accordionshape. Depending on the number of boxes used, it can be a bed or even become, alternatively, a table or a stool.

It can withstand up to $300 \mathrm{~kg}$ in weight (a common foldaway bed in aluminium and technical fabric bears only up to about $125-150 \mathrm{~kg}$ ) and face conditions of up to $75 \%$ humidity.

In addition to greater versatility with respect to a common camp bed, it has other positive facets. It can be produced on site, without the need for specialized manufacturing, thus supporting the local economy and reducing the cost and environmental impact of transport. Moreover, this latter could be done in large quantities on wooden pallets. Once its life cycle is concluded, it can be recycled without producing additional waste and more than that, in an emergency context, it can be used in many other circumstances in which it is expected temporary use.

Education in a Box was a program for the education of children affected by disasters, drawn up by the English charity Education for All. ${ }^{22}$ A normal container filled with educational and emergency supplies, such as tents, furniture, boards, stationery, toys etc., was shipped to affected areas. There, the empty container should have been part of the arrangement of the temporary school prepared with the materials therein contained.

The equipment of one container could satisfy the requirements for up to 200

20. "Moduli ad Uso Scolastico Provvisorio" in Italian.

21. Leaf Supply is a French company engaged in the humanitarian field and in the promotion of sustainable development. It cooperates with national and international agencies and NGOs and it has received several awards for its work. For further information, refer to http://www.leafsupply. com/crbst_0_en.html.

22. Education for All is a charity established in the UK in 2007. It collects disposal material and school furniture to ship them to areas affected by disaster consequences, in order to re-establish or start education programs in different countries. For further information, refer to http://www.edu cationforall.com/Education_for_All/Home.html. 
pupils and may vary according to special educational needs, from the Early Childhood to Adults education. Surely it was a positive example of recovery and reuse that allows postponing the disposal of still usable facilities. Nonetheless, in any case, they were pre-owned materials, which as in good condition, may have a residual duration limited in time, in the face of remarkable efforts for the procurement and transport.

\section{Results}

On the basis of the findings above, a stepwise design method ${ }^{23}$ has been constituted for achieving valuable answers, coherent with the theme under examination and also applicable once the emergency has finished.

In the design approach, the attention mainly focused on the interiors, furnishing and on the setting-up, while with regards to the facility structures, they have selected from time to time, among existing projects, and currently in production.

Thus, in accordance with the considerations arisen during the previous phases of the research, the design process outlined the following, sequential stages:

- guidance for dimensioning, analysis of climate distribution, landscape features of Italian territory and selection of four scenarios of action;

- selection of the outer shells, suitable respectively for each scenario;

- identification of the common design guidelines;

- design of the concepts in the strict sense.

They are briefly described hereafter.

From the Guidance for Dimensioning to the Scenarios of Action

The design hypothesis theorizes to accommodate about 60 children, divided into three classrooms.

UNHCR prescribes to provide one school for 5000 people. $^{24}$ UNICEF indicates a minimum area for temporary school buildings should be 1.2 square metres per child, and to be increased in case of Early Childhood Development programs. $^{25}$

Finally, the International Network for Education in Emergencies (INEE) gives Guidance for designing temporary schools with special attention to the safer school construction and the measures that "must still be taken to ensure that

23. The stepwise design methodology is directly inspired and follows the approach of Bruno Munari, as outlined in B. Munari, Da Cosa Nasce Cosa: Appunti per una Metodologia Progettuale (Rome: Laterza, 1981).

24. See "Table 11 - Typical Services and Infrastructure Requirements for Refugee Camps," in United Nations High Commissioner for Refugees. Handbook for Emergencies, 1982, 553.

25. See United Nations International Children's Emergency Fund, Compendium Temporary Learning Spaces (TLS) - Design and Practice in Emergencies, 2011, 13. 
temporary shelters do not pose a further risk to children and teachers." 26

So, according to this guidance, the proposal concepts have been dimensioned in no less than 90 square metres, calculating that at least 1.5 square metres per child.

Then, the Italian territory has been examined under the profile of the landscape features and the climate distribution, as stated by Wladimir Köppen, in order to select projects which are most suitable to respond to four different situations. These have been selected among the most representative Italian environments, considering that requirements could be influenced in relation to the context where operating.

So, it has been decided to work with three different situations: a coldtempered mountain context, an urban field with Oceanic climate and a rural zone with Hot-summer Mediterranean climate.

\section{Selection of the Outer Shells}

In the next step, through a wide analysis of the newest proposals and most innovative, they have been selected the casings among both those already commercially available or that are still at the prototype stage.

The structures chosen as outer shells should take into account the environmental and climatic characteristics of each context in which it has been hypothesized their use.

\section{Identification of the Design Guidelines}

This phase of the design process has been the most complex of the whole study, as they should have been considered and addressed all the issues previously highlighted in the Methodologies:

- the behaviour and needs of children aged 3-6 and their response to sudden and traumatic events;

- the pivotal role played by the school at the physical, psychological and social level in helping both individuals and communities;

- the normal requirements of preschool and the main critical issues of the temporary structures usually devoted to accommodating activities during emergency situations.

All these aspects have contributed to delineating the guiding principles of the developed design methodology that has further an ideal reference to the educational philosophy of the Reggio Emilia Approach. Its idea of quality, in facts, refers to the school environment and foresees the possibility of fluid and spontaneous enjoyment of the so-called relational space. ${ }^{27}$

26. See Inter-Agency Network for Education in Emergencies (INEE), INEE Guidance Notes on Safer School Construction. Global Facilities for Disaster Reduction and Recovery, 2009, 61.

27. Refer to G. Ceppi and M. Zini, Children, Spaces, Relations: Metaproject for an Environment for Young Children (Milan: Domus Academy Research Center, 1998), 12-13. 
Then, to identify the types of spaces to prepare, the procedure was implemented in two phases. The first one consists of reviewing the activities that are usually carried out at school in normal conditions.

The purpose was to individuate the driving parameters for designing a minimal but complete environment, and being able to satisfy all the requirements, even if in difficulties for space.

At a later stage, evaluated the experts' strategies of intervention in emergencies, they have been compared these two methodologies. Thus, starting from examining the usual organization of kindergarten, broadly speaking, they can be identified three types of areas:

- for ritual moments that mark the school day: "care actions" like coming in and out of school, meals, hygiene, sleep;

- for learning activities: educational activities in the strict sense;

- for free playing and socialization.

In a more detailed classification, the following activities can be considered and organized in specific locations within the school:

- coming in and out: hall, coat hangers or lockers;

- learning activities: at the table, in the classroom;

- free activities: playing, indoors or outdoors (rumpus room or courtyard/ garden);

- meals: snacks and lunch, in the lunchroom;

- hygiene practice: bathroom;

- rest: in the cribs room.

With particular regard to learning activities, some corners inside the classroom are dedicated to a special moment of the day, realizing a subdivision of the unitary space that helps children in identifying and recognizing the several areas. For this, it is possible for the teacher to maintain the possibility of overall control.

It is possible to set corners for different ways of playing that let children improve all their skills and competencies. For example, the games of "Pretending to...", like the puppets, dressing-up or the playhouse, are to heighten socialization, affection and emotion. Motor, graphics and manipulative activities, such as dance or drawing, enhance their body awareness and their knowledge.

Furthermore, books and instruments give to the children the chance to experiment, investigate and deepen their understanding of the world.

Finally, as sometimes it could be hard for children to deal with the absence of parents, or more simply there could be moments of stress during the permanence at school, it's appropriate to provide soft spaces, with rugs and pillows, like "denspaces". There, it could be possible to relax and use it to hide if in need of solitude.

Naturally, all these indications are not to be intended as duress, but rather a path, a suggestion that takes account the right of children to customize the space over time in a gradually appropriating, free use, and open to changes. 
In the second phase, it has been observed an overlap between some school activities and those proposed by psychologists and volunteers during the postcrisis intervention.

It is useful for the victims and helps to cope with the consequences of the experience. In fact, they are particularly suitable for this purpose the expressive and artistic activities, such as drawing, painting, manipulation, but also drama, story-telling and reading of fairy tales, as well as psycho-motor activity. Thanks to these activities, in the hard context of the emergence children can:

- relieve stress caused by old and new sources of fear, as far as possible;

- recognize and express the feelings originated with the new situation;

- share the emotions with friends and adults who take care of them;

- reinforce the relations, enhancing their feelings of belonging to a group, empathy and solidarity.

Therefore, through cross-referencing, we can define what should be the requirements of a state of emergency educational space. In addition to the devices that allow the possibility of satisfying other daily needs, should also be available the following:

- $\quad$ "Pretending to ..." corner for role-playing and drama;

- Book corner for reading and narrative;

- Atelier or other art workshops for drawing, painting, manipulation;

- Den-space and soft corner for intimacy and reassurance exigences.

Thanks to this analysis and to the highlighted elements, it had been possible to outline the common guidelines for the four design concepts, that so have been declined each one in accordance with the theme previously identified, but, in all of them, keeping the application of the same principles.

\section{The Concepts}

In accordance with the considerations explained above, the final step of the design method originated with this research, has been the proposal of four concepts, foreseen for as many different hypotheses of intervention. They share the same underlying stepwise approach, but varying from time to time the choice of the outer shells and their interior settings, depending on the context for which they are envisaged.

They are the pre-cast industrialized house Armadillo, a modular structure with high-efficiency energy performances, briefly called Lègologica and a domeshaped structure realized with the Super-Adobe techniques: they are respectively suitable for the urban area, the mountain context and the rural zones. The tepee tent will complete the framework of the options to move, as an ideal solution for the first-aid response, independent from climate and territorial characteristics. All the design concepts shall be described in detail below. 


\section{A Tepee for the First Emergency Response ${ }^{28}$}

The first design concept deals with the theme of the "first emergency response", to be provided within a period of a few days. The aim is to give a wideranging solution, to be easily adapted to different landscape and climate conditions, and that can be quick to set up on site.

For this, it has been chosen a flexible casing structure, the Tepee. These are tents that are usually utilised as open-air accommodation facilities, exhibition spaces or alternative households for private individuals as leisure spaces.

\section{Technical Characteristics}

Tepees are produced by various companies throughout the world with highperforming materials that ensure very good performances in isolation, waterproof and thermal comfort.

They were chosen the ones that are produced by the Italian-Dutch company Gioielli del bosco - Bosjuweel, which has many years of experience in the manufacture of tepees and yurts.

They are available in a range of dimension between 4.5 and 9 meters, to accommodate a minimum of 9 to a maximum of 70 people sitting. They employ a load-bearing structure of about 17 Douglas wood poles, which are linked at one end to form an inverted cone. Subsequently, the structure is covered with sheeting constituted by a double layer of fabric, of which the external one is waterproof and breathable. While the internal protects from the cold, it also contributes to the rise of the combustion fumes from the central heating fire towards the summit opening that can be suitably adjusted according to the wind direction. Both the fabrics are produced by the Dutch company TenCate and thanks to their breathability, neither can be affected by condense or hackable by mould.

Even if they have never been used in the context of the emergency response, these features make tepees a suitable solution in this field of application, even more so when it is considered that their performances are significantly better than the ones provided by tents currently used by the civil protection department.

Moreover, the tepee for its highly recognizable archetypal shape could have positive effects on the emotional reactions of children, creating an elsewhere, just as a game through which they could deal more lightly with the difficulties of the early stages of the emergence.

\section{Setting}

The hypothesis envisages the use of two tepees 8 metres in diameter, connected to each other for a total covered surface area of slightly more than 120 square metres. Therefore, it is more than sufficient to meet the dimensional requirements.

As a first emergency response, it is intended to remain in site for a maximum of 6 months, the proposed interior solution explores the many opportunities for use

28. See https://bit.ly/2IEKCJ2 or https://bit.ly/2XsgaVV. 
of cardboard, both as regards the partitions that the furnishings.

Smaller areas that are allocated to specific activities could be organized using self-standing screens or dividers made of cardboard, wood or fabric.

For example, the SÉPARÉ that was designed by students Anna Damoli and Chiara Gutierrez, of the Faculty of Design and Art of the Free University of Bolzano, during the workshop Container Village - Living in a box. ${ }^{29}$ Built with a ladder and a carpet, it ensures the flexibility of use, being able to assume different configurations and can be customized by the user.

Furthermore, the Cocoon House (presented by Ophélie Bertout and Marcus Kistner at the Carton Plein! Exhibition) ${ }^{30}$ is helpful for separation or as a little hideaway. It is obtained from a single large sheet of cardboard, broken down and folded along a pattern of diagonal lines. Once put into the form it can be used in various ways, depending on the side on which it is resting.

Finally, two projects of the Argentinian design studio Pomada employ cardboard barrels in the realization of two elements, particularly suitable for this interior project. A partition, that could also be used to contain when necessary, and the stackable Chupito stools, that for their loosely ethnic ornament are particularly in harmony with the character of the tepee (Figure 1).

For the urban context with Oceanic climate, the outer shell chosen is a project that was developed between 2004 and 2005, by the Italian design studio Atelier2. It was called Armadillo, due to the ribs on the extrados of the panel, that recall its namesake animal's armour. The Italian architects Valentina Gallotti and Marco Imperadori, who both worked in the past with the French designers Eric Dubosc and Marc Landowski, had designed a prototype for an industrialized house in close collaboration with a team of architects, engineers and industrials. In order to achieve the maximum standardization of the construction process, but without losing in terms of quality, it is based on the use of readily available materials and equipment. The result is an architecture industrialized, modular and flexible, with ample freedom of customization in the definition of the size and internal partition.

29. Container Village - Living in a box, workshop directed by professors Claudio Larcher and Giampietro Gai. It took place during the summer semester 2013.

30. Carton Plein! exhibition at the Cité de l'architecture à Paris, 03/02 - 28/03/2010. Refer to F. Meadows, Carton Plein!: 13 Architectes à l' Exercice de la Cabane (Paris: Édition Alternatives, 2010). 


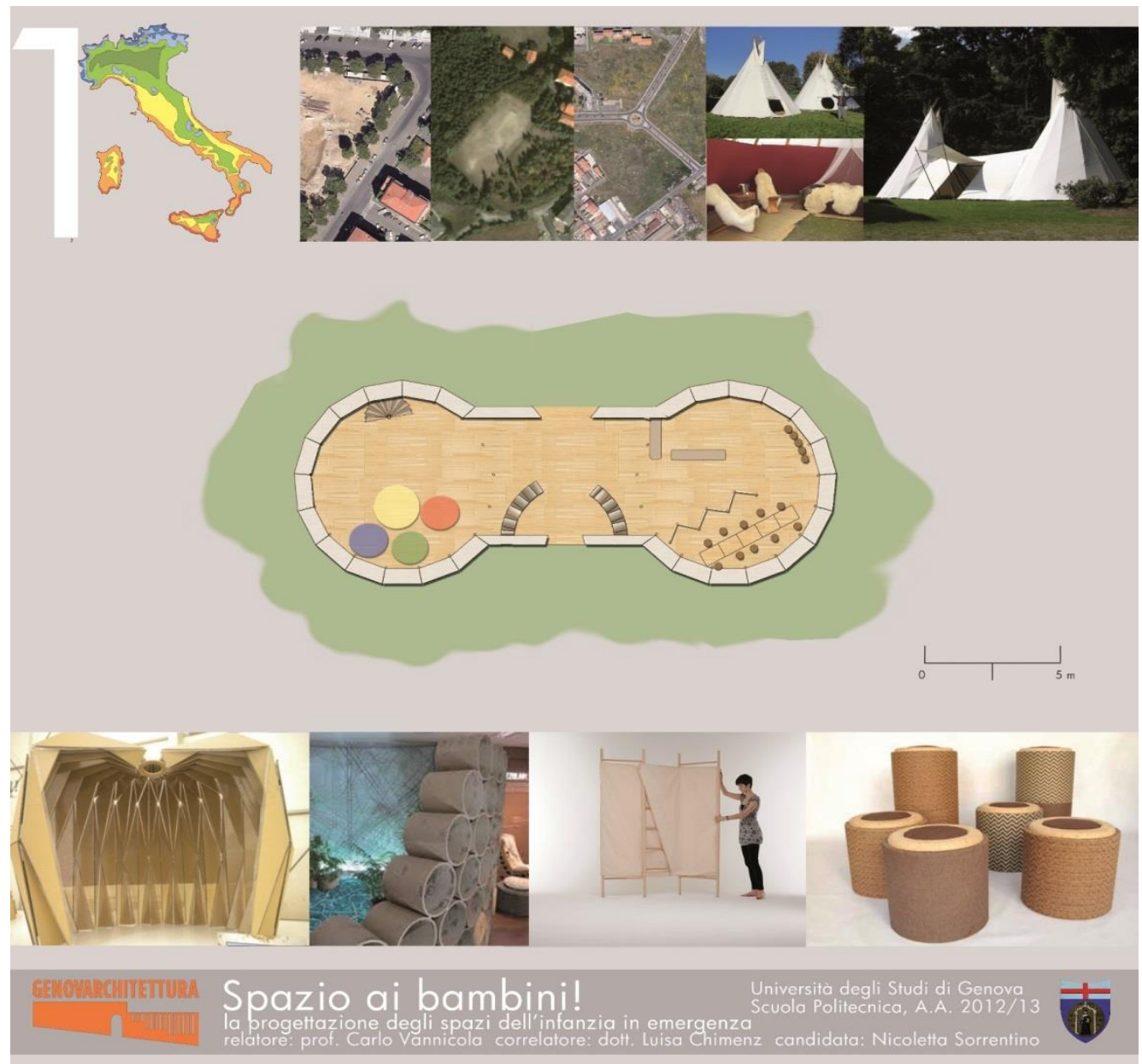

Figure 1. Concept Design for First-Aid Response

"Armadillo": House Prêt-à-Porter for the Urban Context ${ }^{31}$

Initially developed for residential purposes, this project is actually characterized by great versatility which makes it suitable for different intended uses, such as for temporary health or school structures, with a prediction of use in the medium and long term.

\section{Technical Characteristics}

The basic unit consists of a shell with semi-circular cross-section and rectangular plan size, equal to $6.60 \times 8.00$ metres. This is divisible into two subunits from $6.60 \times 4.00$ metres where necessary or can be extended up to a length of 12 or 16 metres. One of the greatest strengths of this shell-like structure is the Elycop curved sandwich panel. Thanks to the inner layer of polyurethane foam of $80 \mathrm{~mm}$, it ensures better performance in terms of isolation, if compared to the panels most widely used in emergency prefab structures.

The modules can also be joined laterally to each other, solving the fitting connection between the two curved surfaces by means of Elyplast flat panels,

31. See http://www.atelier2.it/opere/larmadillo-casa-a-guscio-pret-a-porter/. 
which are produced by the same company. The constructive technology employed in the realization of Armadillo involves the use of a supporting frame, constituted by steel profiles and not calendered, mounted on a foundation that is well anchored into the ground.

On this framework, the Elycop panels are dry assembled and collaborate to its stiffening while they cover the structure. The closures in the header are complemented by means of pre-painted aluminium windows, as well as steel sandwich panels and polyurethane foam. Inside, the frame allows the anchoring of any partition, achievable into a wide range of possibilities, as well as it is available the broader the range of customization of finishing materials. The assembly process is very fast and takes a few days.

\section{Setting}

For this concept, the hypothesis starts from considering an area of about 160 square metres, employing three basic units of $6.60 \times 8.00$ metres, side by side to each other, and connected by access on a balcony.

The theme is the adaptability: thanks to movable and removable furnishing and dividers, it is possible to vary from time to time the spatial configuration, accomplishing the need for more space for movement activities, or separation to create quietness and attention.

In this aim, it has been selected the modular partition system Softwall designed by the Canadian company Molo Design. It is a self-supporting wall made of textile derived from polyester and is $100 \%$ recyclable (trade name Tyvek). It has the characteristics of flexibility and foldability to its honeycomb structure. Available in three different heights and relative thickness, it can be anchored to a wall and kept closed or alternatively it can be opened to create a soft wall. It is deployable at will, able to soften the sound, and to diffuse or absorb light, depending on the colour chosen (black, white or kraft paper).

Regarding the other furnishing to be considered in the setting of Armadillo, they were selected tables and stools of the Muzzle ${ }^{\circledR}$ series by Paratelier, designed by Italian architects Leonardo Paiella and Monica Ravazzolo.

They are made starting from yellow Doka boards used in construction sites, from which are obtained by laser cutting the pieces forming the assembling kit of each element. It will be assembled by means of joints and without the aid of glues or hardware.

To store books and other teaching materials, the Waybasics bookshelves and racks could be located in the classroom. They are produced with the Zboard, which is a special material obtained from pressed cardboard plates that are treated superficially with bright colours. This makes the material more resistant than the ordinary paper board but retaining its lightness and handling characteristics.

Regarding the soft area for the rest, it could be prepared by using mats produced by the Italian company Play+ in close collaboration with Reggio Children. They are part of an integral line of furniture for the kindergarten, developed by applying to the design process the whole know-how of the Reggio Emilia Approach. The mats chosen for the setting of Armadillo can be used on 
both sides for a dual function, having a more rigid and supported side which is suitable for motor activities. The other one, softer and cosy, is usable for the rest.

In an emergency set-up, this feature allows us not to give up the precious moments for the growth of the children because of the possible scarcity of space.

Finally, interpreting the theme of transformation/convertibility under a different point of view, it was decided to include an igloo, built with plastic bottles, as den-space. This item might be a small self-construction that is to be carried out together with the children themselves, with a view to a shared process and to participate in, which would lead to a finished product expression of the collaboration of all. Its positivity would implement in many respects, not only as regards to the mode of realization but also for the added value of the activity as support for overcoming the traumatic situation. In addition, it would constitute an important shared memory, once the situation has come back to normal (Figure 2).

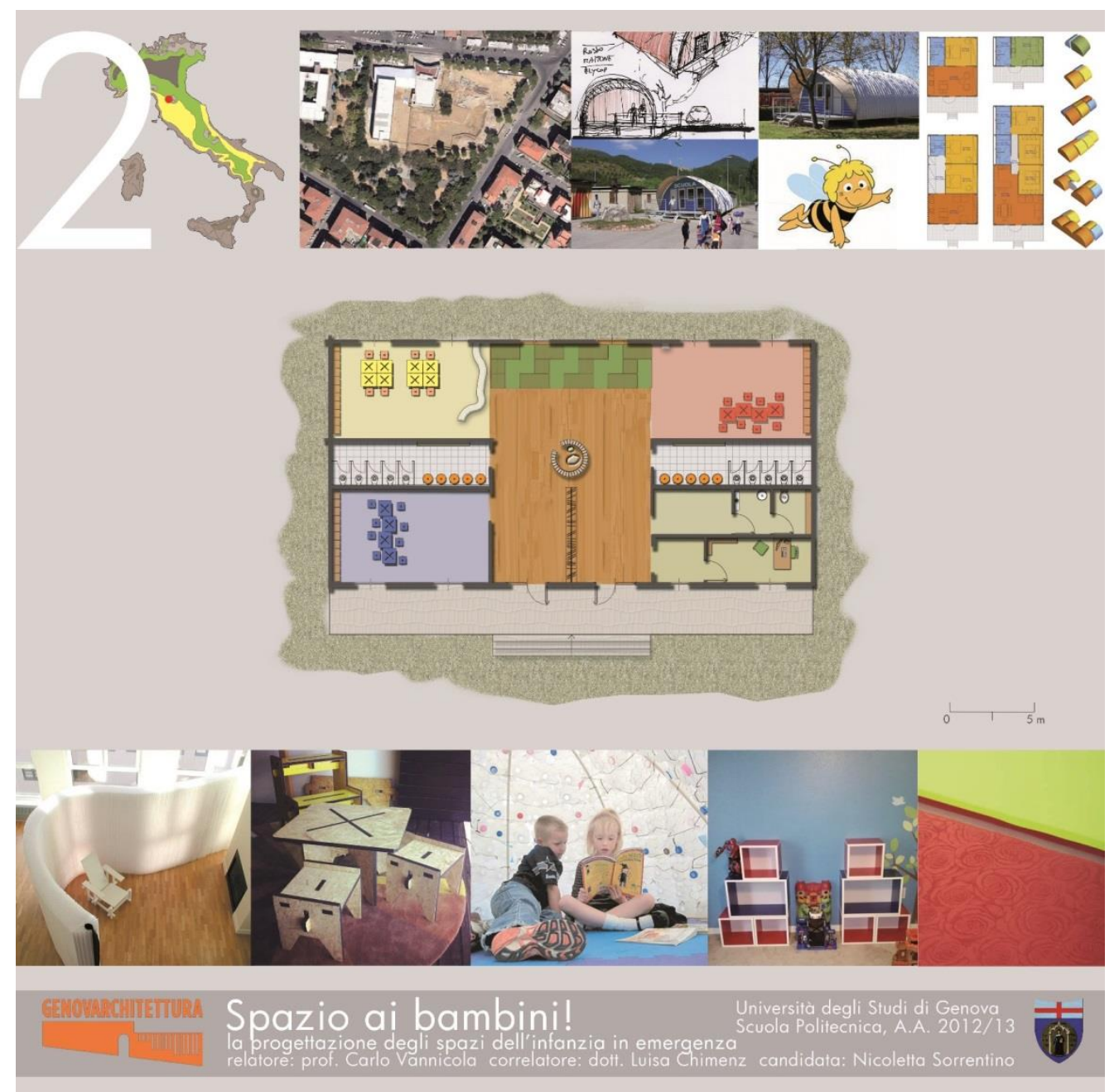

Figure 2. Concept Design for Armadillo 


\section{"Lègologica: A House Full of Content",32}

The structure chosen for the mountain environment is Lègologica, a house full of content and locally sourced, according to the name given to it by the designers, Italian architects Francesco Bombardi and Simone Ardigò.

As the name suggests, it has a double reference to the ecology, due to the peculiarity of being completed with natural materials found on site, and the modularity, which is inspired by the famous Danish game of multicolour bricks.

It was exhibited for the first time in Rome in the summer of 2012, during the exhibition at the MAXXI Museum. Thanks to it, the designers and companies that had participated in the creation of the prototype gained the labelEco_Luoghi 2011, assigned by the Italian Minister of the Environment.

\section{Technical Characteristics}

The basic unit has a size of 12 square metres of net usable internal area. Multiple modules can be combined to be built the layout that best fits the needs of the users. It can be easily transported and assembled thanks to the pre-made building blocks, inspired by the fishing nets. They are to be completed via a selfconstruction process, using natural material recovered on the site directly, such as leaves, stones, barks and so on.

It is completely autonomous from the energy point of view, due to the presence of the solar panels, and guarantees a perfect thermal comfort in all seasons, thanks to a passive cooling system capable of generating cross ventilation.

The mass and weight of the empty brick shells are filled with local materials and provide stability to the structure without the need for invasive foundations.

The building technology is based on a frame consisting of glulam asymmetric portals. On them is fixed the cover, made of photovoltaic panels and which is consequently closed by a wooden infill that is about $15 \mathrm{~cm}$ thick, where can be also housed within the technical installations.

The completion of the structure is entrusted to external blocks of considerable thickness, realized as empty baskets and without load-bearing function. They come to the construction site to be assembled on the spot and filled with materials that are found on site. They have to be provided with particle size and consistency, to ensure high energy efficiency together with the insulating material of the internal infilling.

For the use of natural materials, Lègologica sets itself in dialogue with the surrounding environment, morphing in turn with the seasons. Moreover, thanks to the arrangement of the windows that can be freely decided during the installation, it directly involves the user in an empathetic relation with nature and landscape.

\section{Setting}

Unlike the previous cases, in this one it was necessary to assume the opportunity to apply a different method of connection between the units, imagining

32. See http://www.francescobombardi.it/legologica.html. 
a plan using 14 of them.

Two could be used individually, one as an office, a teacher room and an infirmary, while the other one as a unit of connection between the classrooms. The remaining 12, aggregated in pairs and placed in a flower-shaped arrangement, could be used as classrooms, a rumpus room and a bathroom. This development plan is approximately 170 square metres.

Due to the double temper of Lègologica, which uses natural materials such as wood or leaves and gravel, but is high-tech in terms of building methods, the interior design is played on these features, with key determinant projects mixing nature and artifice.

They are the Fagus tree-shaped bookshelves, produced by the Italian society AL2698; pouffes Livingstones by Smarin that seem like real giant stones until you try their softness. The Puzzle Carpets by Magis Metoo that in their three decorative variants recreate the natural water surface, a lawn or a sandy beach. By the same company, the wire-mesh decoration set Birds+Clouds is suitable for a ceiling fixture. Legno Vivo by Riva1920 that is a collection usable as a coffee-table or stools.

By means of these projects, it could be possible to prepare different areas within the pre-school, creating a relaxing environment and situations that evoke various environmental contexts (Figure 3).

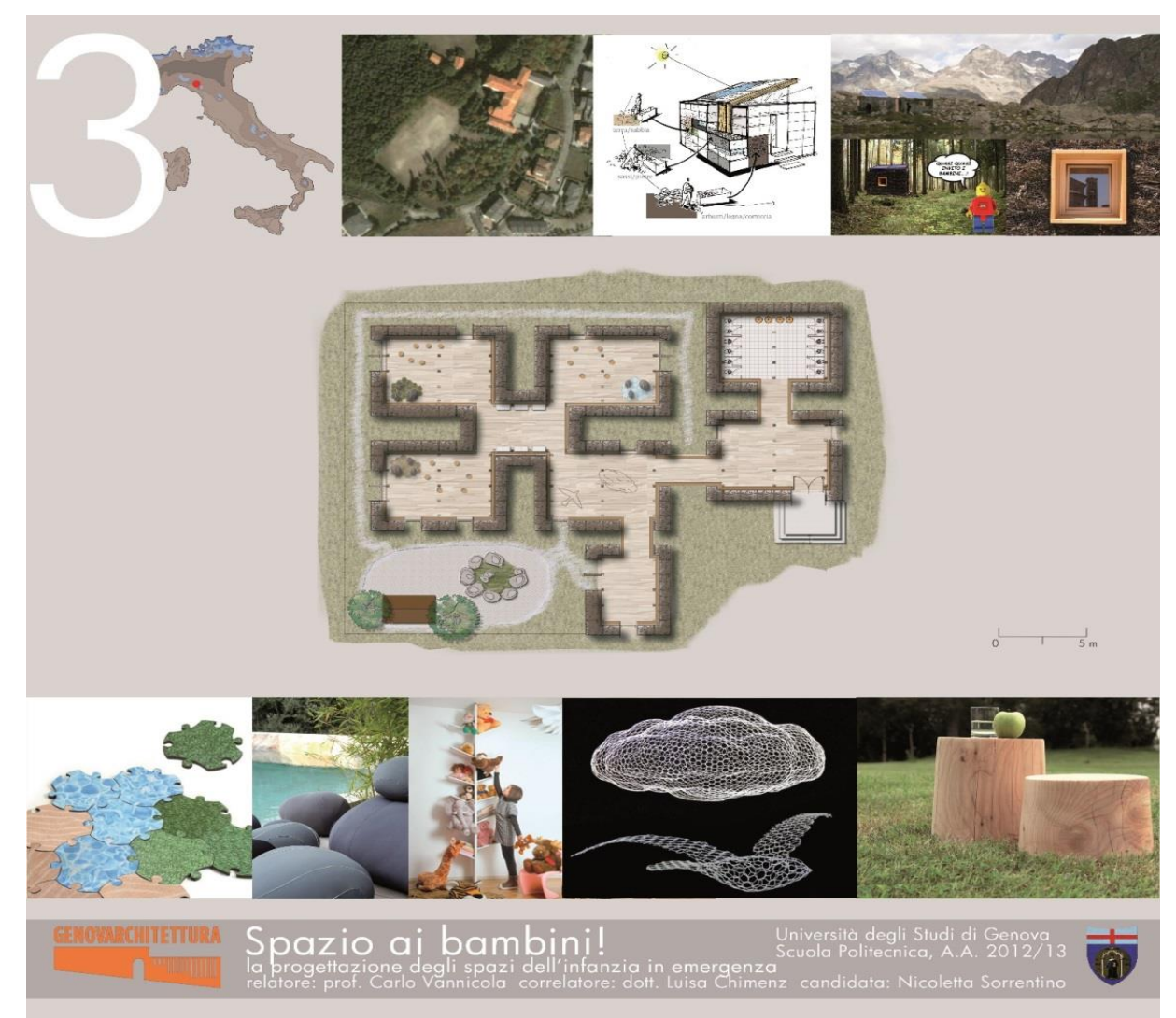

Figure 3. Concept Design for Lègologica 


\section{An Ecological Dome for the Mediterranean Rural Context ${ }^{33}$}

The project chosen for the Mediterranean context with a warm temperate climate (Hot-summer Mediterranean clime, according to Köppen's classification), is the structure made with coiled sandbags developed by Nader Kahlili. This architect of Iranian origin had always had a particular attention to humanitarian issues. In 1991 he founded the California Institute of Earth Art and Architecture (so-called Cal-Earth), where he developed his research on structures, made with Superadobe technology. Thanks to this innovative construction method, based on traditional building techniques, it is possible to build arches, self-supporting domes and vaults without the aid of camber.

It is a simple, economical architecture and is very durable. It is also often used during crisis situations or where the supply of construction materials is particularly difficult. Furthermore, it could be used in the medium-long term, even in unfavourable warm climates, whereas its high thermal inertia looks like a certainly advantageous feature.

\section{Technical Characteristics}

The basic unit is constituted of a central dome surrounded by four semicircular niches; it can reach the size of 37 square metres and it is possible to combine two modules, in order to obtain a larger housing unit, which is slightly lower than 80 square metres. So, to meet the dimensional requirements, it has been designed using four basic units, three of which are to be allocated to the classrooms, while the last one to host service functions as an office, a changing room and the infirmary.

The utilization of solar energy and radiant panels provide heating, as well as the thickness of the sandbag walls contributes to the maintenance of the indoor thermal comfort with high energy efficiency.

Furthermore, this particular building process consent to integrate in-progress the insertion of masonry, by exploiting the four semi-circular niches on the perimeter. Thus, it is possible to liberate the internal space from clutter, to increase fluidity and functional use.

\section{The Setting}

Whereas this concept revolves around the theme of a rural context with a warm temperate climate, the project proposal deliberately puts on hold the organization of interior spaces and it completely devotes its attention to what happens outside the facilities.

Once arranged the four domes around a central court, the project develops the organization of the resulting spaces delimited by the fluid shapes of the four building units, at the centre of the court as well as on the perimeter.

As previously considered, the outdoor activities are of particular importance for children in normal conditions, and still more to facilitate the overcoming of

33. See http://www.calearth.org/superadobe-structures-calearth. 
traumatic events. Consistent with the possible consequences for the region, this project has tried to draw some opportunities for experiences outside the school, without limit them only to the classic playground or sports field, but placing other points of interests for children.

First of them takes its inspiration from The Maternelle sous Chapiteau, a project developed in 2008 by Zoom Architecture studio with a kindergarten class in Calais. During a workshop dedicated to introducing children to the circus arts, it was included the construction of a small pavilion with lycra and coloured cardboard tubes, ridden by children themselves.

In this case, it may be interpreted as the creation of an atelier, within which to gather experimental activities of observation and manipulation or become a sort of small "museum" in which to collect and preserve materials and equipment.

In the organization of the external spaces between the domes, a small area is also provided to be used as a teaching garden. The cultivation and care of plants, in fact, are meaningful experiences for children and can be a valuable aid in stress mitigation in the case of difficult situations such as emergencies.

The participation of the whole group allows the growth of a sense of belonging in children, helping them to strengthen relations and to have again confidence in life and in the future.

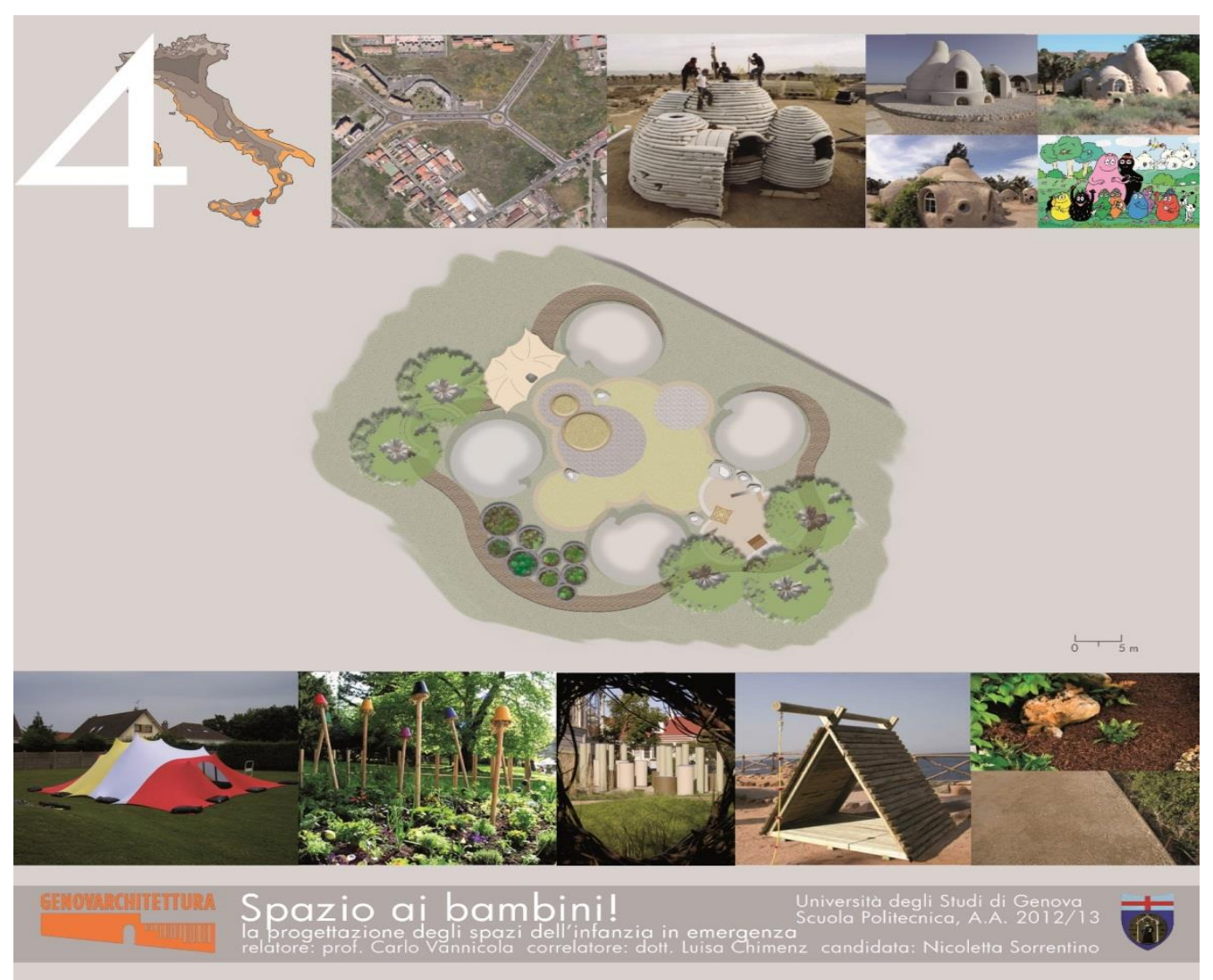

Figure 4. Concept Design for Eco-Dome

It also might be possible to prepare corners for open-air reading and establish a mini outdoor library, where cherish books about natural and scientific themes. 
Eventually, these places could be arranged also as areas for relaxing in the shade of the trees.

Finally, the insertion of a playground is provided as a free zone in which different playing modes, such as climbing, jumping, sliding and swinging could be interpreted in freedom, by means of an organization that follows the natural orientation of the ground.

The paving of the court should be treated with materials of different colours and grain, playing on the development of curves and circular lines and delimiting heterogeneous portions for contours and dimensions. In this way, they could constitute a further and stimulating means for children to make richer their sensory experience (Figure 4).

\section{Conclusions}

Since its early beginning, the problem appeared composite and multifaceted: in fact, as discussed above, many components are to be evaluated when talking about children and their special needs. Moreover, considering the extraordinary circumstances that can affect people and communities after any kind of disaster.

The design process outlined in the research follows a logical and rational path, in order to try bridging the distance between the operational language of the designer and the one sometimes excessively theoretical of institutional guidelines.

Thus, the main intention of this research was not to reach a properly defined architectural design, but rather to outline design suggestions, through which bust the myth of a design, that uses "simplicity" as an excuse, concealing inadequacy and shortage of proposals.

The main objection could be that the examples presented are referred to the Italian context only, so focusing on a necessarily limited area. Moreover, it would be in any case a privileged context, compared to other ones clearly more problematic.

Actually, this instance is a direct consequence of architecture and design, that by their nature require to be contextualized, and cut on a framework of demands more or less restricted; also, by reason of the economic affordability.

However, the concept outlined is only an expression that could have been different simply by means of a choice of diverse housing structures and other furniture settings. The most important aspect lies in the possibility of an integrated design process and interdisciplinary, that it could be adaptable to different situations and gradable in each case, according to particular needs or limitations.

As the problems related to emergencies seem to grow and spread more and more widely, it should be responsibility of designers contributing to mitigate their effects, by offering the necessary competence for improvement in terms of quality, both functional and aesthetic.

The purpose of architecture is to protect and improve humankind's life on earth, so as to satisfy its belief in the nobility of its existence. (Eliel Saarinen) 


\section{Bibliography}

Bosi, R. La Cura Nella Scuola dell' Infanzia. [The Care in the Preschool.] Rome: Carocci, 2007.

Ceppi, G. and M. Zini. Children, Spaces, Relations: Metaproject for an Environment for Young Children. Milan: Domus Academy Research Center, 1998.

Giller, E. What is Psychological Trauma. Brooklandville, MD: Sidran Institute, 1999.

Global Protection Cluster. Guidelines for Child-Friendly Spaces in Emergencies. Global Education Cluster, INEE \& IASC, 2011.

Inter-Agency Network for Education in Emergencies (INEE). INEE Guidance Notes on Safer School Construction. Global Facilities for Disaster Reduction and Recovery. 2009.

Iori, V. Lo Spazio Vissuto: Luoghi Educative e Soggettività. [The Experienced Space: Educative Places and Subjectiveness.] Florence: Nuova Italia, 1996.

Isidori, M. V. "Principali Criticità della Pedagogia e della Didattica dell' Emergenza." [Main Critical Issues of Pedagogy and Didactics in Emergency.] Studi sulla Formazione 13, no. 1 (2010): 133.

Isidori, M. V. and A. Vaccarelli. Pedagogia dell' Emergenza/Didattica nell' Emergenza. I Processi Formativi nelle Situazioni di Criticità Individuali e Collettive. [Pedagogy of the Emergency/Didactics in Emergency. Formative Processes in Individual and Collective Critical Situations.] Milan: Franco Angeli, 2013.

Leaning, J. and G. S. Debarati. "Natural Disasters, Armed Conflict, and Public Health." New England Journal of Medicine 369, no. 19 (2013): 1836-1842.

Lo Iacono, G. and L. Ranzato. "Aiutare i Bambini Sopravvissuti a Calamità: Indicazioni per Insegnanti e Genitori." [Help Children Survivors of Disasters: Guidance for Teachers and Parents.] Psicologia e Psicologi I, no. 3 (2001).

Meadows, F. Carton Plein!: 13 Architectes à l' Exercice de la Cabane. [Carton Full!:13 Architects at the Exercise of the Hut.] Paris: Édition Alternatives, 2010.

Munari, B. Da Cosa Nasce Cosa: Appunti per una Metodologia Progettuale. [One Thing Leads to Another: Notes for a Design Methodology.] Rome: Laterza, 1981.

United Nations High Commissioner for Refugees. Handbook for Emergencies, 1982.

United Nations International Children's Emergency Fund. Compendium Temporary Learning Spaces (TLS) - Design and Practice in Emergencies, 2011. 
